\title{
Valuing equity-linked death benefits in jump diffusion models
}

\author{
Hans U. Gerber*, Elias S. W. Shiuł Hailiang Yang ${ }^{\ddagger}$
}

\begin{abstract}
The paper is motivated by the valuation problem of Guaranteed Minimum Death Benefits in various equity-linked products. At the time of death, a benefit payment is due. It may depend not only on the price of a stock or stock fund at that time, but also on prior prices. The problem is to calculate the expected discounted value of the benefit payment. Because the distribution of the time of death can be approximated by a combination of exponential distributions, it suffices to solve the problem for an exponentially distributed time of death. The stock price process is assumed to be the exponential of a Brownian motion plus an independent compound Poisson process whose upward and downward jumps are modeled by combinations (or mixtures) of exponential distributions. Results for exponential stopping of a Lévy process are used to derive a series of closed-form formulas for call, put, lookback, and barrier options, dynamic fund protection, and dynamic withdrawal benefit with guarantee. We also discuss how barrier options can be used to model lapses and surrenders.
\end{abstract}

Key words: Equity-linked death benefits, variable annuities, jump diffusion, exponential stopping, barrier options.

JEL Classification: G13 G22 C02

Subject Categories: $\quad$ IM10 IE50 IM40 IB10

\section{Introduction}

This paper is a continuation of Gerber, Shiu and Yang (2012). The motivation is the problem of valuing Guaranteed Minimum Death Benefits (GMDB) in various variable annuity and equity-indexed annuity contracts. Our goal is to present actuaries with a methodology that they can use to value and reserve for such guarantees.

*Department of Statistics and Actuarial Science, The University of Hong Kong, and Faculty of Business and Economics, University of Lausanne, CH-1015 Lausanne, Switzerland, e-mail: hgerber@unil.ch.

${ }^{\dagger}$ Department of Statistics and Actuarial Science, The University of Iowa, Iowa City, Iowa 52242-1409, U.S.A., e-mail: elias-shiu@uiowa.edu

$\ddagger$ Department of Statistics and Actuarial Science, The University of Hong Kong, Pokfulam Road, Hong Kong, e-mail: hlyang@hku.hk. 
In Gerber, Shiu and Yang (2012), the price of a stock or stock fund is modeled as a geometric Brownian motion, i.e., the price at time $t$ is

$$
S(t)=S(0) e^{X(t)}, \quad t \geq 0,
$$

where $\{X(t) ; t \geq 0\}$ is a Brownian motion or Wiener process. In this paper, we generalize $\{X(t)\}$ as a jump diffusion, i.e., a Brownian motion plus an independent compound Poisson process. For actuaries, the use of compound Poisson processes can be traced back to the 1903 doctoral thesis of the Swedish actuary Filip Lundberg. In Lundberg's collective risk theory, aggregate claims are modeled by compound Poisson processes. The family of compound Poisson processes is rich in that it is dense in the family of all Lévy processes, of which jump diffusions are special cases. Therefore, the addition of an independent Brownian motion was not a big step and, understandably, has not found wide publicity in the actuarial literature. Two early papers are Gerber $(1970,1972)$. The title of Dufresne and Gerber (1991b), "Risk theory for the compound Poisson process that is perturbed by diffusion," is indicative of the actuarial perception of the jump diffusion model.

In finance, the jump diffusion model is considered from a substantially different perspective. The economic significance of modeling stock price movements by geometric Brownian motion was recognized by Samuelson (1965). For modeling jumps in stock prices, Merton (1976) added an independent compound Poisson process to the Brownian motion. This was an important advance, as geometric Brownian motion models do not account for empirical facts such as heavy tails and volatility smiles. An excellent survey of jump diffusion models in finance can be found in Kou (2008), who and whose co-authors have done pioneering work in this field.

Jump diffusions are particularly tractable if the distribution of the jumps is a combination (or a mixture) of exponential distributions. In actuarial science, it is found that if the individual claim distribution is modeled as a combination (or a mixture) of exponential distributions, closed-form expressions for the probability of ruin, for the expected discounted dividends until ruin, and other quantities of interest can be readily determined. See, for example, Täcklind (1942), Dufresne and Gerber (1988, 1989, 1991a, 1991b), Chan (1990), Gerber and Shiu (1998, 2005), Chan, Gerber and Shiu (2006), Gerber, Shiu and Smith (2006), Avanzi and Gerber (2008), and so on.

Consider a variable annuity for a person now age $x$. There is a GMDB rider that guarantees the following payment to his estate when he dies,

$$
\max \left(S\left(T_{x}\right), K\right)
$$

where $T_{x}$ is the time-until-death random variable and $K$ is the guaranteed minimum amount. Since

$$
\max \left(S\left(T_{x}\right), K\right)=S\left(T_{x}\right)+\left[K-S\left(T_{x}\right)\right]_{+},
$$

the problem of valuing the guarantee becomes the problem of valuing a $K$-strike put option that is exercised at the time of death. Because policy surrenders or lapses should also be incorporated in the valuation model, the problem is to determine the following expectation:

$$
\mathrm{E}\left[e^{-\delta T_{x}} e^{-\theta T_{x}} \pi\left(S(0) e^{M\left(T_{x}\right)}\right)\left[K-S\left(T_{x}\right)\right]_{+}\right]
$$


Here, $\delta$ denotes a continuously compounded valuation interest rate (valuation force of interest). $M\left(T_{x}\right)$ is the maximum of $\{X(t)\}$ up to $T_{x}$; because of $(1.1), S(0) e^{M\left(T_{x}\right)}$ is the maximum price of a unit of the stock fund between time 0 and $T_{x}$. The function $\pi(s)$ is 1 for $s \leq S(0)$, and it is a nonnegative and nonincreasing function of $s$ for $s>S(0)$; the function $\pi(s)$ is to capture the phenomenon that the higher is the stock price, the less valuable is the guarantee (put option), and hence the higher is the tendency for policyholders to surrender their policies. The exponential function $e^{-\theta t}$, with $\theta$ being a positive constant, models that independent of the stock fund performance, a constant proportion of remaining policies will lapse in each subsequent time period. The product of the two factors, $e^{-\theta t} \pi\left(S(0) e^{M(t)}\right)$, gives the fraction of in-force policies at time $t$.

By considering the valuation force of interest to be $(\delta+\theta)$, we can ignore the factor $e^{-\theta T_{x}}$ in (1.4). Thus we are motivated to examine expectations of the form

$$
\mathrm{E}\left[e^{-\delta T_{x}} g_{T_{x}}(S)\right]
$$

where $g_{t}(S)$ is a functional of the stock price process up to time $t$. For the case

$$
g_{t}(S)=\pi\left(S(0) e^{M(t)}\right)[K-S(t)]_{+},
$$

we seem to need to find the joint probability density function (pdf) $f_{X\left(T_{x}\right), M\left(T_{x}\right)}(x, y)$ for evaluating (1.5).

Here is a summary of our approach. (I) The distribution of the positive random variable $T_{x}$ can be approximated by linear combinations of exponential distributions. Then, under the assumption that $T_{x}$ is independent of the stock price process $\{S(t)\}$, the problem of approximating the expectation (1.5) reduces to that of evaluating

$$
\mathrm{E}\left[e^{-\delta \tau} g_{\tau}(S)\right]
$$

where $\tau$ is an arbitrary exponential random variable independent of $\{S(t)\}$. (II) We can use the factorization,

$$
\begin{aligned}
\mathrm{E}\left[e^{-\delta \tau} g_{\tau}(S)\right] & =\mathrm{E}\left[e^{-\delta \tau}\right] \mathrm{E}^{*}\left[g_{\tau}(S)\right] \\
& =\frac{\lambda}{\lambda+\delta} \mathrm{E}^{*}\left[g_{\tau}(S)\right]
\end{aligned}
$$

to take care of the discount factor. Here, $\lambda$ is the parameter of $\tau$, i.e., $\mathrm{E}[\tau]=1 / \lambda$. The asterisk signifies that the parameter of $\tau$ is changed to $\lambda+\delta$, i.e., $\mathrm{E}^{*}[\tau]=1 /(\lambda+\delta)$. Hence, this paper will derive formulas for

$$
\mathrm{E}\left[g_{\tau}(S)\right]
$$

not (1.7). (III) Let $M(\tau)$ denote the running maximum of the Lévy process $\{X(t)\}$ up to time $\tau$. The random variables $M(\tau)$ and $[X(\tau)-M(\tau)]$ are independent. Hence the joint pdf of $X(\tau)$ and $M(\tau)$ can be factorized,

$$
f_{X(\tau), M(\tau)}(x, y)=f_{M(\tau)}(y) \times f_{X(\tau)-M(\tau)}(x-y), \quad \max (x, 0) \leq y .
$$

To determine the two pdf's on the right-hand side (RHS) of (1.10), we find their moment generating functions $(\mathrm{mfg})$ by means of the identity

$$
\mathrm{E}\left[e^{z X(\tau)}\right]=\mathrm{E}\left[e^{z M(\tau)}\right] \times \mathrm{E}\left[e^{z[X(\tau)-M(\tau)]}\right] .
$$


Details of this important step are given in Section 3.

For readers interested in Laplace transforms, we note the following two facts. Consider the expectation

$$
\mathrm{E}\left[g_{t}(S)\right]
$$

as a function of $t, t \geq 0$; the Laplace transform of (1.12) with respect to the parameter $\lambda$ is $\mathrm{E}\left[g_{\tau}(S)\right] / \lambda$. Consider the surplus process defined by

$$
U(t)=u-X(t), \quad t \geq 0
$$

the Laplace transform of the time of ruin random variable with respect to the parameter $\lambda$ is the probability $\operatorname{Pr}(M(\tau) \geq u)$.

We consider options that are exercised at time $\tau$. In Sections 4 to 8, we derive formulas for valuing various call, put, lookback, and barrier options. Section 9 values "dynamic fund protection" when the guarantee is effective until time $\tau$. Section 10 considers the dual concept of "dynamic withdrawal benefit" and values a put option on the residual account value exercised at time $\tau$.

\section{Exponential stopping of a Lévy process}

In this section we set up a general framework. More specific results will be given in subsequent sections.

Let $\{X(t) ; t \geq 0\}$ be a Lévy process. We assume that the moment generating function (mgf) of $X(t)$ exists in some nonempty interval. Then the mgf of $X(t)$ is

$$
\mathrm{E}\left[e^{z X(t)}\right]=e^{t \Psi(z)}
$$

where $\Psi(z)$ denotes the Lévy exponent of the process, that is, the cumulant generating function of $X(1)$. The Lévy process is stopped at time $\tau$, an exponential random variable with mean $1 / \lambda$ that is independent of the Lévy process. We are interested in finding the distribution of $X(\tau)$. By conditioning on $\tau$, we find a first answer in terms of the mgf of $X(\tau)$ :

$$
\mathrm{E}\left[e^{z X(\tau)}\right]=\mathrm{E}\left[\mathrm{E}\left[e^{z X(\tau)} \mid \tau\right]\right]=\mathrm{E}\left[e^{\tau \Psi(z)}\right]=\frac{\lambda}{\lambda-\Psi(z)}
$$

With (2.2) we may be able to identify the distribution of $X(\tau)$.

The domain of definition of $\Psi(z)$ can be extended by means of analytic continuation. Even though it is an abuse of notation, we shall use $\Psi(z)$ to denote such functions. Suppose that $\Psi(z)$ is a rational function and that the roots of

$$
\Psi(z)=\lambda
$$


are distinct. Let $\left\{\alpha_{j}\right\}$ and $\left\{\beta_{k}\right\}$ be the roots with negative and positive real part, respectively. Equation (2.3) has no roots with zero real part because for each purely imaginary number $z$,

$$
\left|\mathrm{E}\left[e^{z X(\tau)}\right]\right| \leq \mathrm{E}\left[\left|e^{z X(\tau)}\right|\right]=\mathrm{E}[1]=1
$$

Since

$$
\frac{\lambda}{\lambda-\Psi(z)}=\sum_{j} \frac{\lambda}{\Psi^{\prime}\left(\alpha_{j}\right)} \frac{1}{\alpha_{j}-z}+\sum_{k} \frac{\lambda}{\Psi^{\prime}\left(\beta_{k}\right)} \frac{1}{\beta_{k}-z},
$$

the pdf of $X(\tau)$ is

$$
f_{X(\tau)}(x)= \begin{cases}\sum_{j} a_{j} e^{-\alpha_{j} x}, & \text { if } x<0, \\ \sum_{k} b_{k} e^{-\beta_{k} x}, & \text { if } x \geq 0\end{cases}
$$

where

$$
a_{j}=\frac{-\lambda}{\Psi^{\prime}\left(\alpha_{j}\right)}
$$

and

$$
b_{k}=\frac{\lambda}{\Psi^{\prime}\left(\beta_{k}\right)}
$$

For a Lévy process with a diffusion component, the pdf $(2.5)$ is continuous at $x=0$. This can be seen as follows. If the left-hand side (LHS) of (2.4) is written as a ratio of two polynomials, the degree of the denominator is at least two higher than that of the numerator. It follows that the sum of all residues vanishes, that is,

$$
\sum_{j} \frac{\lambda}{-\Psi^{\prime}\left(\alpha_{j}\right)}+\sum_{k} \frac{\lambda}{-\Psi^{\prime}\left(\beta_{k}\right)}=0
$$

which is indeed equivalent to the continuity of $(2.5)$ at $x=0$.

Let $M(t)$ denote the running maximum of the Lévy process, and $m(t)$ the running minimum. We are also interested in $M(\tau)$ and $m(\tau)$, the observed extrema until time $\tau$.

In this paper, the Lévy process $\{X(t) ; t \geq 0\}$ is usually a Brownian motion (with drift and diffusion parameters $\mu$ and $\sigma$ ) extended by independent jumps in both directions. The downward jumps form an independent compound Poisson process; the frequency of these jumps is $\nu$. Similarly, the upward jumps forms another independent compound Poisson process with Poisson parameter $\omega$.

Let us assume that the pdf of each downward jump is

$$
\sum_{j=1}^{m} A_{j} v_{j} e^{-v_{j} x}, \quad x>0
$$

with $\sum_{j=1}^{m} A_{j}=1$ and $0<v_{1}<v_{2}<\ldots<v_{m}$, and that the pdf of each upward jump is

$$
\sum_{k=1}^{n} B_{k} w_{k} e^{-w_{k} x}, \quad x>0
$$


with $\sum_{k=1}^{n} B_{k}=1$ and $0<w_{1}<w_{2}<\ldots<w_{n}$. Then

$$
\Psi(z)=D z^{2}+\mu z-\nu \sum_{j=1}^{m} A_{j} \frac{z}{v_{j}+z}+\omega \sum_{k=1}^{n} B_{k} \frac{z}{w_{k}-z},
$$

where

$$
D=\sigma^{2} / 2
$$

Under the assumption that the $m+n+2$ solutions of the equation (2.3) are distinct, the density function of $X(\tau)$ is given by (2.5). In the case of mixtures (all $A_{i}^{\prime} s$ and $B_{i}^{\prime} s$ positive), the solutions are distinct and real as we have the following interlacing relationship:

$$
-\infty<\alpha_{m+1}<-v_{m}<\ldots<-v_{1}<\alpha_{1}<0<\beta_{1}<w_{1}<\ldots<w_{n}<\beta_{n+1}<\infty
$$

A figure similar to Figure 13.6.2 of Bowers et al. (1997) can be helpful in visualizing (2.13). The unlikely situation that some of the solutions coincide can occur only in the case of combinations (some of the $A_{i}^{\prime} s$ or $B_{i}^{\prime} s$ negative). In the case of combinations, the $m+n+2$ solutions are definitely distinct if $\lambda$ is sufficiently large; see Theorem 3.1 of Cai and Kou (2011). Note that this also follows from the following asymptotic formulas for $\lambda \rightarrow \infty$ :

$$
\begin{aligned}
\alpha_{m+1} & \approx-\sqrt{\frac{\lambda}{D}}, \quad \beta_{n+1} \approx \sqrt{\frac{\lambda}{D}}, \\
\alpha_{j} & \approx-v_{j}\left[1-\operatorname{sign}\left(A_{j}\right) \frac{\nu}{\nu+\lambda}\right], \quad j=1,2, \ldots, m, \\
\beta_{k} & \approx w_{k}\left[1-\operatorname{sign}\left(B_{k}\right) \frac{\omega}{\omega+\lambda}\right], \quad k=1,2, \ldots, n .
\end{aligned}
$$

Remark 2.1: For the jump diffusion model given in the last paragraph, exactly $m+1$ solutions of equation (2.3) have negative real part, and $n+1$ solutions have positive real part. We have shown that there are no solutions with zero real part. Thus this follows from a continuity argument by starting with (2.13) or (2.14).

Remark 2.2: With $m=n=1,\{X(t)\}$ may be called a double exponential jumpdiffusion. In finance, the term "double exponential jump-diffusion" may mean the resulting stock price process $\{S(t)\}$, and the term "Kou's model" is also used in recognition of the work of S.G. Kou of Columbia University. In this paper, the term "Kou's model" is used to describe both $\{X(t)\}$ and the associated $\{S(t)\}$. For formulas in Kou's model, we shall write $v$ for $v_{1}$ and $w$ for $w_{1}$. In particular, the interlacing relation (2.13) becomes

$$
-\infty<\alpha_{2}<-v<\alpha_{1}<0<\beta_{1}<w<\beta_{2}<\infty
$$

For the purpose of actuarial valuation, Kou's model seems quite sufficient.

Remark 2.3: Instead of (2.9) and (2.10), some researchers would model the jumps with phase-type distributions or mixtures of Erlang distributions. It is known (e.g., Ko 
and $\mathrm{Ng} 2007$ ) that combinations of exponential distributions are also a family of distributions that is weakly dense in the space of all probability distributions on the positive axis. Hence, in the limit, these three families of distributions yield equivalent results. We refer those readers interested in formulas arising from modeling the jumps with phase-type distributions to Asmussen, Avram and Pistorius (2004) and Mordecki (2002).

Remark 2.4: Some authors, such as Asmussen, Avram and Pistorius (2004), call equation (2.3) a Cramér-Lundberg equation. Let $I_{(.)}$denote the indicator function. The condition for which the stochastic process

$$
\left\{e^{z X(t)} I_{(t<\tau)} ; t \geq 0\right\}
$$

becomes a martingale is

$$
\mathrm{E}\left[e^{z X(t)}\right] e^{-\lambda t}=1
$$

which means that $z$ is a solution of $(2.3)$.

\section{Consequences of independence of $M(\tau)$ and $[M(\tau)-$ $X(\tau)]$}

A key tool for finding the distributions of $M(\tau)$ and $m(\tau)$ is the fact that the random variables $M(\tau)$ and $[M(\tau)-X(\tau)]$ are independent. An intuitive explanation for this is that the conditional distribution of $[M(\tau)-X(\tau)]$, given $M(\tau)$, cannot possibly depend on $M(\tau)$. Technical proofs and ramifications of this result can be found in books such as Bertoin (1996, Chapter 6), Doney (2007, Chapter 4), and Kyprianou (2006, Chapter 6).

It immediately follows from this independence property that

$$
\mathrm{E}\left[e^{z X(\tau)}\right]=\mathrm{E}\left[e^{z M(\tau)}\right] \mathrm{E}\left[e^{z[X(\tau)-M(\tau)]}\right]
$$

Now, for each fixed $t$,

$$
\begin{aligned}
X(t)-M(t) & =X(t)-\max \{X(s) ; 0 \leq s \leq t\} \\
& =X(t)+\min \{-X(s) ; 0 \leq s \leq t\} \\
& =\min \{X(t)-X(s) ; 0 \leq s \leq t\} .
\end{aligned}
$$

By the stationary property of a Lévy process, the random variables $[X(t)-X(s)]$ and $X(t-s)$ have the same distribution. Hence, $[X(t)-M(t)]$ and $m(t)$ have the same distribution, and $t$ can be replaced $\tau$ because this property is true for all $t$. Consequently, (3.1) can be rewritten as

$$
\mathrm{E}\left[e^{z X(\tau)}\right]=\mathrm{E}\left[e^{z M(\tau)}\right] \mathrm{E}\left[e^{z m(\tau)}\right]
$$

This elegant formula is a version of the celebrated Wiener-Hopf factorization.

Define

$$
M_{X(\tau)}(z)=\frac{\lambda}{\lambda-\Psi(z)}
$$


$M_{X(\tau)}(z)$ is the mgf $\mathrm{E}\left[e^{z X(\tau)}\right]$ when the expectation exists. Note that the poles of $\Psi(z)$ are the zeros of $M_{X(\tau)}(z)$, and the zeros of the denominator (the solutions of equation (2.3)) are the poles of $M_{X(\tau)}(z)$. Specifically, the zeros of $M_{X(\tau)}(z)$ are $-v_{1}, \ldots,-v_{m}$, which are $m$ negative numbers, and $w_{1}, \ldots, w_{n}, n$ positive numbers; the poles of $M_{X(\tau)}(z)$ are $\alpha_{1}, \ldots, \alpha_{m+1},(m+1)$ numbers with negative real part, and $\beta_{1}, \ldots, \beta_{n+1},(n+1)$ numbers with positive real part. Because $0 \leq M(\tau)<\infty$, the mgf $\mathrm{E}\left[e^{z M(\tau)}\right]$ is an analytic function of $z$ with negative real part and it has no negative zeros. Similarly, the mgf $\mathrm{E}\left[e^{z m(\tau)}\right]$ is an analytic function of $z$ with positive real part and it has no positive zeros. Therefore, from the factorization formula (3.2), we obtain the following pair of proportional relations:

$$
\begin{aligned}
& \mathrm{E}\left[e^{z m(\tau)}\right] \propto\left[\prod_{j=1}^{m}\left(z+v_{j}\right)\right]\left[\prod_{j=1}^{m+1} \frac{1}{z-\alpha_{j}}\right], \\
& \mathrm{E}\left[e^{z M(\tau)}\right] \propto\left[\prod_{k=1}^{n}\left(z-w_{k}\right)\right]\left[\prod_{k=1}^{n+1} \frac{1}{z-\beta_{k}}\right] .
\end{aligned}
$$

As each mgf takes the value 1 when $z=0$, we have

$$
\begin{aligned}
\mathrm{E}\left[e^{z m(\tau)}\right] & =\left[\prod_{j=1}^{m} \frac{z+v_{j}}{v_{j}}\right]\left[\prod_{j=1}^{m+1} \frac{-\alpha_{j}}{z-\alpha_{j}}\right], \\
\mathrm{E}\left[e^{z M(\tau)}\right] & =\left[\prod_{k=1}^{n} \frac{w_{k}-z}{w_{k}}\right]\left[\prod_{k=1}^{n+1} \frac{\beta_{k}}{\beta_{k}-z}\right] .
\end{aligned}
$$

With distinct $\left\{\beta_{j}\right\}$, the partial fraction expansion of the RHS of (3.5) is

$$
\sum_{k=1}^{n+1} \frac{b_{k}^{*}}{\beta_{k}-z},
$$

where

$$
b_{k}^{*}=\left[\prod_{i=1}^{n} \frac{w_{i}-\beta_{k}}{w_{i}}\right]\left[\prod_{i=1, i \neq k}^{n+1} \frac{\beta_{i}}{\beta_{i}-\beta_{k}}\right] \beta_{k} .
$$

Hence, the pdf of $M(\tau)$ is

$$
f_{M(\tau)}(x)=\sum_{k=1}^{n+1} b_{k}^{*} e^{-\beta_{k} x}, \quad x>0 .
$$

Similarly, we apply the method of partial fractions to (3.4) to obtain the pdf of $m(\tau)$,

$$
f_{m(\tau)}(x)=\sum_{j=1}^{m+1} a_{j}^{*} e^{-\alpha_{j} x}, \quad x<0,
$$

where

$$
a_{j}^{*}=\left[\prod_{i=1}^{m} \frac{\alpha_{j}+v_{i}}{v_{i}}\right]\left[\prod_{i=1, i \neq j}^{m+1} \frac{-\alpha_{i}}{\alpha_{j}-\alpha_{i}}\right]\left(-\alpha_{j}\right) .
$$


We can give alternative expressions for the coefficients $a_{j}$ and $b_{j}$ introduced in Section 2. Analogous to (3.3), let the RHS of (3.5) be denoted as $M_{M(\tau)}(z)$ and that of (3.4) be $M_{m(\tau)}(z)$. It then follows from

$$
M_{X(\tau)}(z)=M_{M(\tau)}(z) M_{m(\tau)}(z)
$$

that

$$
a_{j}=a_{j}^{*} M_{M(\tau)}\left(\alpha_{j}\right)
$$

and

$$
b_{k}=b_{k}^{*} M_{m(\tau)}\left(\beta_{k}\right)
$$

We now find the joint densities $f_{X(\tau), M(\tau)}(x, y)$ and $f_{X(\tau), m(\tau)}(x, y)$. Because $M(\tau)$ and $[M(\tau)-X(\tau)]$ are independent, we have, for $y \geq \max (x, 0)$,

$$
\begin{aligned}
f_{X(\tau), M(\tau)}(x, y) & =f_{M(\tau), X(\tau)-M(\tau)}(y, x-y) \\
& =f_{M(\tau)}(y) f_{X(\tau)-M(\tau)}(x-y) \\
& =f_{M(\tau)}(y) f_{m(\tau)}(x-y) \\
& =\left[\sum_{k=1}^{n+1} b_{k}^{*} e^{-\beta_{k} y}\right]\left[\sum_{j=1}^{m+1} a_{j}^{*} e^{-\alpha_{j}(x-y)}\right] \\
& =\sum_{j=1}^{m+1} \sum_{k=1}^{n+1} a_{j}^{*} b_{k}^{*} e^{-\left(\beta_{k}-\alpha_{j}\right) y-\alpha_{j} x} .
\end{aligned}
$$

Similarly, for $y \leq \min (x, 0)$,

$$
f_{X(\tau), m(\tau)}(x, y)=\sum_{j=1}^{m+1} \sum_{k=1}^{n+1} a_{j}^{*} b_{k}^{*} e^{-\left(\alpha_{j}-\beta_{k}\right) y-\beta_{k} x} .
$$

As a check, let us integrate the RHS of (3.14) to see that we can recover the pdf's $f_{m(\tau)}(y)$ and $f_{X(\tau)}(x)$. Note that we have

$$
M_{M(\tau)}(z)=\sum_{k=1}^{n+1} \frac{b_{k}^{*}}{\beta_{k}-z} .
$$

Integrating the RHS of (3.14) with respect to $x$ from $y$ to $\infty$, applying (3.15) with $z=0$, and noting that $M_{M(\tau)}(0)=1$ yields the RHS of (3.8) with $x$ replaced by $y$. Integrating (3.14) with respect to $y$ from $-\infty$ to $x$, applying (3.15) with $z=\alpha_{j}$, and noting (3.11) yields the upper expression on the RHS of (2.5).

Remark 3.1: There are other methods, without explicitly using the fact that $M(\tau)$ and $[X(\tau)-M(\tau)]$ are independent, to derive a formula for the distribution of $M(\tau)$. One approach can be found in Cai and Kou (2011). Some actuaries may prefer the following 
derivation in the context of ruin theory. For $t \geq 0$, let the surplus of a company at time $t$ be

$$
U(t)=u-X(t)
$$

where $u$ is a positive number representing the initial surplus. Let

$$
T=\inf \{t: U(t) \leq 0\}
$$

be the time of ruin. Then,

$$
\operatorname{Pr}(M(\tau) \geq u)=\operatorname{Pr}(\tau>T)=\mathrm{E}\left[e^{-\lambda T}\right] .
$$

Thus, knowing the Laplace transform, with respect to $\lambda$, of the time of ruin random variable is equivalent to knowing the distribution of the $M(\tau)$. In particular, if $\{X(t)\}$ is a Lévy process with an upward jump pdf of the form (2.10), we can use the results in Section 9 of Albrecher, Gerber and Yang (2010), with $w(x) \equiv 1$ and $w_{0}=1$, to obtain a closed-form expression for $\operatorname{Pr}(M(\tau) \geq u)$. Note that in this ruin-theory approach, it is not necessary that the downward jump pdf is of the form (2.9). Also, the relation (3.18) can be refined. For example, let $\Pi_{d}(u)$ denote the probability that the process $\{X(t)\}$ reaches $u$ before time $\tau$ and the first time this occurs, it is because of oscillation. Then

$$
\Pi_{d}(u)=\mathrm{E}\left[e^{-\lambda T} I_{(U(T)=0)}\right]
$$

Now, we set $w(x) \equiv 0$ and $w_{0}=1$ in Section 9 of Albrecher, Gerber and Yang (2010).

Remark 3.2: As pointed out in the last remark, the discounted penalty function approach to deriving a formula for the distribution of $M(\tau)$ does not require specifying the downward jump distribution. Likewise, formula (3.7) remains valid for an arbitrary downward jump distribution. Here, $\beta_{1}, \beta_{2}, \ldots, \beta_{n+1}$ are those solutions, with positive real part, of the following Cramér-Lundberg equation:

$$
D z^{2}+\mu z-\nu \int_{0}^{\infty}\left(1-e^{-z x}\right) \phi_{d}(x) \mathrm{d} x+\omega \sum_{k=1}^{n} B_{k} \frac{z}{w_{k}-z}=0,
$$

with $\phi_{d}(x)$ denoting the pdf of the downward jumps. See also Mordecki (2002). Similarly, formula (3.8) holds for an arbitrary upward jump distribution with $\alpha_{1}, \alpha_{2}, \ldots, \alpha_{m+1}$ being those solutions, with negative real part, of the equation,

$$
D z^{2}+\mu z-\nu \sum_{j=1}^{m} A_{j} \frac{z}{v_{j}+z}+\omega \int_{0}^{\infty}\left(e^{z x}-1\right) \phi_{u}(x) \mathrm{d} x=0,
$$

where $\phi_{u}(x)$ is the pdf of the upward jumps.

Remark 3.3: In Kou's model $(m=n=1)$,

$$
f_{M(\tau)}(x)=\frac{\beta_{2}\left(w-\beta_{1}\right)}{w\left(\beta_{2}-\beta_{1}\right)} \beta_{1} e^{-\beta_{1} x}+\frac{\beta_{1}\left(\beta_{2}-w\right)}{w\left(\beta_{2}-\beta_{1}\right)} \beta_{2} e^{-\beta_{2} x}, \quad x \geq 0,
$$

and

$$
f_{m(\tau)}(x)=\frac{-\alpha_{2}\left(\alpha_{1}+v\right)}{v\left(\alpha_{1}-\alpha_{2}\right)}\left(-\alpha_{1} e^{-\alpha_{1} x}\right)+\frac{\alpha_{1}\left(v+\alpha_{2}\right)}{v\left(\alpha_{1}-\alpha_{2}\right)}\left(-\alpha_{2} e^{-\alpha_{2} x}\right), \quad x \leq 0
$$




\section{Call and put options}

In the following, the price of one unit of a stock (or of a stock fund) at time $t$ is denoted as $S(t)$. We assume

$$
S(t)=S(0) e^{X(t)}, \quad t \geq 0 .
$$

The pdf $f_{X(\tau)}(x)$ is given by (2.5). We consider a contract that is defined by a benefit function $b(s)$ : it provides a payment of $b(S(\tau))$ at time $\tau$. The benefit function for a $K$-strike put option is $b(s)=(K-s)_{+}$, and the one for a $K$-strike call is $b(s)=(s-K)_{+}$.

We are interested in the expected discounted value of the benefit payment,

$$
\begin{aligned}
V(\delta, \lambda) & =\mathrm{E}\left[e^{-\delta \tau} b(S(\tau))\right] \\
& =\lambda \int_{0}^{\infty} e^{-(\lambda+\delta) t} \mathrm{E}[b(S(t))] d t .
\end{aligned}
$$

Here $\delta$ is an appropriate continuously compounded rate of interest. It follows from (4.2) that

$$
V(\delta, \lambda)=\frac{\lambda}{\lambda+h} V(\delta-h, \lambda+h)
$$

for any $h>-\lambda$. In particular, for $h=\delta$,

$$
V(\delta, \lambda)=\frac{\lambda}{\lambda+\delta} V(0, \lambda+\delta) .
$$

This identity shows that it suffices to have formulas and results for $V(0, \lambda)$, the time- 0 expectation of the benefit payment. Then, the time- 0 expected discounted value of the payment, $V(\delta, \lambda)$, is obtained from the substitution $\lambda \leftarrow \lambda+\delta$ and multiplication by $\frac{\lambda}{\lambda+\delta}$. We note that the values of $\alpha_{j}$ 's and $\beta_{k}$ 's will be modified, because the substitution should also be made in the denominator of (2.2) or, equivalently, in equation (2.3).

From now on, we assume the interest rate is 0 . Setting $\delta=0$ in (4.2), we have

$$
V(0, \lambda)=\int_{-\infty}^{\infty} b\left(S(0) e^{x}\right) f_{X(\tau)}(x) d x
$$

where $f_{X(\tau)}(x)$ is given by (2.5) in conjunction with (2.6) - (2.7) or (3.11) - (3.12). For $b(s)=s$,

$$
V(0, \lambda)=\mathrm{E}[S(\tau)]=S(0) \frac{\lambda}{\lambda-\Psi(1)}
$$

by (2.2). The existence of the expectation requires the condition

$$
\Psi(1)<\lambda .
$$

Consider the notation

$$
\eta(h ; K)=\frac{S(0)^{h} K^{1-h}}{h(h-1)}
$$


where $K$ is a positive number and $h$ is a complex number. In this paper, this arises in two situations. (i) For $S(0) \geq K$ and the real part of $h, \operatorname{Re}(h)$, is negative,

$$
\int_{-\infty}^{0}\left[K-S(0) e^{x}\right]_{+} e^{-h x} d x=\int_{-\infty}^{\ln [K / S(0)]}\left[K-S(0) e^{x}\right] e^{-h x} d x=\eta(h ; K) .
$$

(ii) For $S(0) \leq K$ and $\operatorname{Re}(h)>1$,

$$
\int_{0}^{\infty}\left[S(0) e^{x}-K\right]_{+} e^{-h x} d x=\int_{\ln [K / S(0)]}^{\infty}\left[S(0) e^{x}-K\right] e^{-h x} d x=\eta(h ; K) .
$$

Thus it immediately follows from (2.5) that we have the following time- 0 expectations for options that are out-of-the money (or at-the-money). For the put,

$$
\mathrm{E}\left[[K-S(\tau)]_{+}\right]=\sum_{j=1}^{m+1} a_{j} \eta\left(\alpha_{j} ; K\right)
$$

since $S(0) \geq K$, and for the call,

$$
\mathrm{E}\left[[S(\tau)-K]_{+}\right]=\sum_{k=1}^{n+1} b_{k} \eta\left(\beta_{k} ; K\right)
$$

since $S(0) \leq K$. Note that for the expectation on the LHS of (4.12) to exist, we need condition (4.7).

To obtain the in-the-money formulas, we use put-call parity (for $\delta=0$ ). From the identity

$$
[K-S(\tau)]_{+}-[S(\tau)-K]_{+}=K-S(\tau)
$$

it follows that

$$
\mathrm{E}\left[[K-S(\tau)]_{+}\right]-\mathrm{E}\left[[S(\tau)-K]_{+}\right]=K-\mathrm{E}[S(\tau)],
$$

where the expectation $\mathrm{E}[S(\tau)]$ can be found in (4.6). Hence, the in-the-money time-0 expected values are

$$
\mathrm{E}\left[[K-S(\tau)]_{+}\right]=\sum_{k=1}^{n+1} b_{k} \eta\left(\beta_{k} ; K\right)+K-\mathrm{E}[S(\tau)]
$$

for the put $(S(0)<K)$, and

$$
\mathrm{E}\left[[S(\tau)-K]_{+}\right]=\sum_{j=1}^{m+1} a_{j} \eta\left(\alpha_{j} ; K\right)-K+\mathrm{E}[S(\tau)]
$$

for the call $(S(0)>K)$.

Remark 4.1: Formula (4.3) can be viewed as a factorization,

$$
\mathrm{E}\left[e^{-\delta \tau} b(S(\tau))\right]=\mathrm{E}\left[e^{-h \tau}\right] \mathrm{E}^{*}\left[e^{-(\delta-h) \tau} b(S(\tau))\right],
$$

where the asterisk signifies that the mean of the exponential random variable $\tau$ is changed to $1 /(\lambda+h)$. The factorization (4.17) remains valid if $\tau$ is a gamma random variable; $\tau$ in the last expectation has an altered scale parameter. 


\section{$5 \quad$ Fixed-strike lookback call option}

In this and the next several sections, we study the expected discounted values of benefit payments that depend on the running maximum or minimum of the stock prices up to time $\tau$. For $t>0$, let $g_{t}(S)$ denote a functional of the stock price process up to time $t$. Equation (4.2) can be generalized as

$$
\mathrm{E}\left[e^{-\delta \tau} b\left(g_{\tau}(S)\right)\right]=\lambda \int_{0}^{\infty} e^{-(\lambda+\delta) t} \mathrm{E}\left[b\left(g_{t}(S)\right)\right] d t,
$$

which means that (4.3), (4.4) and (4.17) can be extended to the case where the benefit payment is a functional of the stock price process up to time $\tau$. Hence, same as the last section, this and the next sections only present formulas for the expectation of benefit payments, that is, for the case $\delta=0$.

The time- $\tau$ payoff of a fixed-strike lookback call option is

$$
\left[\max \left(H, \max _{0 \leq t \leq \tau} S(t)\right)-K\right]_{+}=\left[\max \left(H, S(0) e^{M(\tau)}\right)-K\right]_{+} .
$$

Here, $H$ is a positive constant with $H \geq S(0)$; it can be interpreted as the maximum level of the stock's historical $(t \leq 0)$ prices. To value this payoff, we need to distinguish whether the strike price $K$ is higher or lower than the historical maximum price $H$, that is, we need to distinguish whether the option is out-of-the money or in-the-money.

\section{Out-of-the-money fixed-strike lookback call option}

For $K>H$, the payoff (5.2) simplifies as

$$
\left[S(0) e^{M(\tau)}-K\right]_{+}
$$

whose time- 0 expectation is

$$
\int_{0}^{\infty}\left[S(0) e^{x}-K\right]_{+} f_{M(\tau)}(x) \mathrm{d} x=\sum_{k=1}^{n+1} b_{k}^{*} \eta\left(\beta_{k} ; K\right)
$$

by (3.7) and (4.10).

\section{In-the-money fixed-strike lookback call option}

For $K<H$, the payoff $(5.2)$ is

$$
\max \left(H, S(0) e^{M(\tau)}\right)-K .
$$

By rewriting (5.5) as

$$
H-K+\left[S(0) e^{M(\tau)}-H\right]_{+}
$$

and using (5.4) with $K$ replaced by $H$, we find that the time- 0 expectation of $(5.5)$ is

$$
H-K+\sum_{k=1}^{n+1} b_{k}^{*} \eta\left(\beta_{k} ; H\right) .
$$




\section{Fixed-strike lookback put option}

The time- $\tau$ payoff of a fixed-strike lookbackput option is

$$
\left[K-\min \left(H, \min _{0 \leq t \leq \tau} S(t)\right)\right]_{+}=\left[K-\min \left(H, S(0) e^{m(\tau)}\right)\right]_{+} \cdot
$$

Here, $H$ is a positive constant, with $H \leq S(0)$; it can be interpreted as the minimum level of the stock's historical $(t<0)$ prices. To value this payoff, we need to distinguish whether the strike price $K$ is lower or higher than the historical minimum price $H$, that is, we need to distinguish whether the option is out-of-the money or in-the-money.

\section{Out-of-the-money fixed-strike lookback put option}

For $K<H$, the payoff (6.1) simplifies as

$$
\left[K-S(0) e^{m(\tau)}\right]_{+}
$$

whose time- 0 expectation is

$$
\int_{-\infty}^{0}\left[K-S(0) e^{x}\right]_{+} f_{m(\tau)}(x) \mathrm{d} x=\sum_{j=1}^{m+1} a_{j}^{*} \eta\left(\alpha_{j} ; K\right)
$$

by (3.8) and (4.9).

\section{In-the-money fixed-strike lookback put option}

For $K>H$, the payoff $(6.1)$ is

$$
K-\min \left(H, S(0) e^{m(\tau)}\right)=K-H+\left[H-S(0) e^{m(\tau)}\right]_{+},
$$

whose time- 0 expectation is

$$
K-H+\sum_{j=1}^{m+1} a_{j}^{*} \eta\left(\alpha_{j} ; H\right)
$$

by applying (6.3) with $K$ replaced by $H$.

\section{Floating-strike lookback option}

\section{Floating-strike lookback put option}

The payoff at time $\tau$ is

$$
\max \left(H, \max _{0 \leq t \leq \tau} S(t)\right)-S(\tau),
$$

where $H \geq S(0)$. By comparing (7.1) with (5.5), we see that its time-0 expectation is (5.7) but with $K$ replaced by $\mathrm{E}[S(\tau)]$. The result is

$$
H-\mathrm{E}[S(\tau)]+\sum_{j=1}^{n+1} b_{k}^{*} \eta\left(\beta_{j} ; H\right)
$$


with $\mathrm{E}[S(\tau)]$ is given in (4.6).

\section{Fractional floating-strike lookback put option}

The payoff at time $\tau$ is

$$
\left[\gamma \max _{0 \leq t \leq \tau} S(t)-S(\tau)\right]_{+}
$$

where $\gamma$ is a constant between 0 and 1 . Obviously,

$$
\left[\gamma e^{M(\tau)}-e^{X(\tau)}\right]_{+}=e^{M(\tau)}\left[\gamma-e^{X(\tau)-M(\tau)}\right]_{+} .
$$

Because the random variables $M(\tau)$ and $[X(\tau)-M(\tau)]$ are independent,

$$
\begin{aligned}
& \mathrm{E}\left[\left[\gamma \max _{0 \leq t \leq \tau} S(t)-S(\tau)\right]_{+}\right] \\
& =\mathrm{E}\left[e^{M(\tau)}\right] \mathrm{E}\left[\left[\gamma S(0)-S(0) e^{X(\tau)-M(\tau)}\right]_{+}\right] \\
& =M_{M(\tau)}(1) \mathrm{E}\left[\left[\gamma S(0)-S(0) e^{m(\tau)}\right]_{+}\right] \\
& =M_{M(\tau)}(1) \sum_{j=1}^{m+1} a_{j}^{*} \eta\left(\alpha_{j} ; \gamma S(0)\right)
\end{aligned}
$$

by (6.3). The factor $M_{M(\tau)}(1)$ can be determined using (3.5). In Kou's model, (7.5) is

$$
\frac{\lambda}{D} \frac{w-1}{\left(\beta_{1}-1\right)\left(\beta_{2}-1\right)\left(\alpha_{1}-\alpha_{2}\right)}\left[\left(\alpha_{1}+v\right) \eta\left(\alpha_{1}, \gamma S(0)\right)-\left(v+\alpha_{2}\right) \eta\left(\alpha_{2}, \gamma S(0)\right)\right] .
$$

\section{Floating-strike lookback call option}

The payoff at time $\tau$ is

$$
S(\tau)-\min \left(H, \min _{0 \leq t \leq \tau} S(t)\right)
$$

where $0<H \leq S(0)$. Its time-0 expectation is (6.5) with $K$ replaced by $\mathrm{E}[S(\tau)]$, namely,

$$
\mathrm{E}[S(\tau)]-H+\sum_{j=1}^{m+1} a_{j}^{*} \eta\left(\alpha_{j} ; H\right) .
$$

\section{Fractional floating-strike lookback call option}

The payoff at time $\tau$ is

$$
\left[S(\tau)-\gamma \min _{0 \leq t \leq \tau} S(t)\right]_{+}
$$

where $\gamma \geq 1$ is a constant. Its time-0 expectation is analogous to (7.5),

$$
M_{m(\tau)}(1) \sum_{k=1}^{n+1} b_{k}^{*} \eta\left(\beta_{k} ; \gamma S(0)\right) \text {, }
$$


which, in Kou's model, is

$$
\frac{\lambda}{D} \frac{v+1}{\left(1-\alpha_{1}\right)\left(1-\alpha_{2}\right)\left(\beta_{2}-\beta_{1}\right)}\left[\left(w-\beta_{1}\right) \eta\left(\beta_{1} ; \gamma S(0)\right)+\left(\beta_{2}-w\right) \eta\left(\beta_{2} ; \gamma S(0)\right)\right] .
$$

\section{High-low option}

The high-low option is also called the length-of-range option. Its payoff at time $\tau$ is

$$
\max \left(\bar{H}, \max _{0 \leq t \leq \tau} S(t)\right)-\min \left(\underline{H}, \min _{0 \leq t \leq \tau} S(t)\right),
$$

where $0<\underline{H} \leq S(0) \leq \bar{H}$. The parameters $\underline{H}$ and $\bar{H}$ can be interpreted as the past stock-price minimum and maximum, respectively. We note that the payoff (7.12) is the sum of (7.1) with $H=\bar{H}$ and (7.7) with $H=\underline{H}$. Hence it follows from (7.2) and (7.8) that the time-0 expectation of the high-low option is

$$
\bar{H}+\sum_{k=1}^{n+1} b_{k}^{*} \eta\left(\beta_{k} ; \bar{H}\right)-\underline{H}+\sum_{j=1}^{m+1} a_{j}^{*} \eta\left(\alpha_{j} ; \underline{H}\right) .
$$

\section{Barrier options}

A barrier option is an option whose payoff depends on whether or not the price of the underlying asset has breached a predetermined level or barrier L. Knock-out options are those which go out of existence if the stock price breaches the barrier, and knockin options are those which come into existence if the barrier is breached. We have the following parity relation for the payoffs and hence their expectations:

Knock-out option + Knock-in option = Ordinary option.

Consider $S(0)<L$. Let

$$
\ell=\ln [L / S(0)]
$$

Then $\ell>0$. The payoff of an up-and-in option is

$$
I_{\left(\left[\max _{0 \leq t \leq \tau} S(t)\right] \geq L\right)} b(S(\tau))=I_{(M(\tau) \geq \ell)} b\left(S(0) e^{X(\tau)}\right) .
$$

Its expectation is

$$
\int_{-\infty}^{\ell}\left[\int_{\ell}^{\infty} b\left(S(0) e^{x}\right) f_{X(\tau), M(\tau)}(x, y) \mathrm{d} y\right] \mathrm{d} x+\int_{\ell}^{\infty} b\left(S(0) e^{x}\right) f_{X(\tau)}(x) \mathrm{d} x .
$$

By (3.13), (8.2) and (2.5), this is

$$
\begin{aligned}
& \sum_{j=1}^{m+1} \sum_{k=1}^{n+1} a_{j}^{*} b_{k}^{*} \frac{1}{\beta_{k}-\alpha_{j}}\left[\frac{S(0)}{L}\right]^{\beta_{k}-\alpha_{j}} \int_{-\infty}^{\ell} b\left(S(0) e^{x}\right) e^{-\alpha_{j} x} \mathrm{~d} x \\
& +\sum_{k=1}^{n+1} b_{k} \int_{\ell}^{\infty} b\left(S(0) e^{x}\right) e^{-\beta_{k} x} \mathrm{~d} x \\
& =\sum_{j=1}^{m+1} \sum_{k=1}^{n+1} a_{j}^{*} b_{k}^{*} \frac{1}{\beta_{k}-\alpha_{j}}\left[\frac{S(0)}{L}\right]^{\beta_{k}} \Lambda\left(\alpha_{j}\right)+\sum_{k=1}^{n+1} b_{k}\left[\frac{S(0)}{L}\right]^{\beta_{k}} \Lambda\left(\beta_{k}\right),
\end{aligned}
$$


where

$$
\Lambda(h)= \begin{cases}\int_{-\infty}^{0} b\left(L e^{x}\right) e^{-h x} \mathrm{~d} x & \text { if } \operatorname{Re}(h)<0 \\ \text { undefined } & \text { if } \operatorname{Re}(h)=0 \\ \int_{0}^{\infty} b\left(L e^{x}\right) e^{-h x} \mathrm{~d} x & \text { if } \operatorname{Re}(h)>0 .\end{cases}
$$

Note that $\Lambda(h)$ depends on the function $b(s)$ and the level $L$. Also, if $S(0)=L(\ell=0)$, then the iterated integral in (8.4) simplifies as

$$
\int_{-\infty}^{0} b\left(L e^{x}\right) f_{X(\tau)}(x) \mathrm{d} x
$$

while the first sum on the RHS of (8.5) simplifies as

$$
\sum_{j=1}^{m+1} a_{j}^{*} M_{M(\tau)}\left(\alpha_{j}\right) \Lambda\left(\alpha_{j}\right)=\sum_{j=1}^{m+1} a_{j} \Lambda\left(\alpha_{j}\right),
$$

by (3.15) and (3.11).

Next, consider $S(0)>L(\ell<0)$. The payoff of a down-and-in option is

$$
I_{\left(\left[\min _{0 \leq t \leq \tau} S(t)\right] \leq L\right)} b(S(\tau))=I_{(m(\tau) \leq \ell)} b\left(S(0) e^{X(\tau)}\right) .
$$

Its expectation is

$$
\int_{-\infty}^{\ell} b\left(S(0) e^{x}\right) f_{X(\tau)}(x) \mathrm{d} x+\int_{\ell}^{\infty}\left[\int_{-\infty}^{\ell} b\left(S(0) e^{x}\right) f_{X(\tau), m(\tau)}(x, y) \mathrm{d} y\right] \mathrm{d} x .
$$

By (2.5), (8.2), (3.14) and (8.6), this is

$$
\sum_{j=1}^{m+1} a_{j}\left[\frac{L}{S(0)}\right]^{-\alpha_{j}} \Lambda\left(\alpha_{j}\right)+\sum_{j=1}^{m+1} \sum_{k=1}^{n+1} a_{j}^{*} b_{k}^{*} \frac{1}{\beta_{k}-\alpha_{j}}\left[\frac{L}{S(0)}\right]^{-\alpha_{j}} \Lambda\left(\beta_{k}\right) .
$$

We now give formulas for $\Lambda(h)$ for (knock-in) call and put options. With $S(0)$ replaced by $L$, formulas (4.8) to (4.10) are useful here. Also, we can use the identity

$$
\left(L e^{x}-K\right)_{+}-\left(K-L e^{x}\right)_{+}=L e^{x}-K .
$$

For call options, $b(s)=(s-K)_{+}$, we have

$$
\Lambda(\alpha)= \begin{cases}0 & \text { if } L<K \\ \frac{L^{\alpha} K^{1-\alpha}}{\alpha(\alpha-1)}+\left(\frac{L}{-\alpha+1}-\frac{K}{-\alpha}\right) & \text { if } L>K\end{cases}
$$

and

$$
\Lambda(\beta)= \begin{cases}\frac{L^{\beta} K^{1-\beta}}{\beta(\beta-1)} & \text { if } L<K \\ \frac{L}{\beta-1}-\frac{K}{\beta} & \text { if } L>K\end{cases}
$$


where $\operatorname{Re}(\alpha)<0$ and $\operatorname{Re}(\beta)>0$. For put options, $b(s)=(K-s)_{+}$, we have

$$
\Lambda(\alpha)= \begin{cases}\frac{K}{-\alpha}-\frac{L}{-\alpha+1} & \text { if } L<K \\ \frac{L^{\alpha} K^{1-\alpha}}{\alpha(\alpha-1)} & \text { if } L>K\end{cases}
$$

and

$$
\Lambda(\beta)= \begin{cases}\frac{L^{\beta} K^{1-\beta}}{\beta(\beta-1)}-\left(\frac{L}{\beta-1}-\frac{K}{\beta}\right) & \text { if } L<K \\ 0 & \text { if } L>K\end{cases}
$$

For knock-out options, we can use the identity (8.1). Note that each of the knock-in formulas (8.10) to (8.13) has two expressions, depending on whether $K<L$ or $K>L$. Each ordinary put or call option formula also has two expressions, depending on whether $K<S(0)$ or $K>S(0)$; see the paragraph on in-the-money formulas near the end of Section 4. Because up-and-out options have the initial condition $S(0)<L$, and downand-out options have the initial condition $S(0)>L$, each knock-out put/call formula has three expressions.

Barrier options can be used to model lapses and surrenders. For example, the $\pi(s)$ function in (1.4) can be modeled as

$$
\pi(s)=\sum_{j} p_{j} I_{\left(s<L_{j}\right)}
$$

where $S(0)<L_{1}<L_{2}<L_{3}<\ldots$ and $p_{1}, p_{2}, p_{3}, \ldots$ are positive numbers that sum to one. Thus, if the maximum of the stock price from time 0 and time $t$ is between level $L_{k}$ and level $L_{k+1}$, the proportion of polices still in force at time $t$ is

$$
e^{-\theta t} \sum_{j \geq k+1} p_{j}
$$

\section{Dynamic fund protection}

Here, $S(t)=S(0) e^{X(t)}$ denotes the price of one unit of a mutual fund at time $t \geq 0$. Consider an investor purchasing one unit of the mutual fund at time 0 , together with the following "dynamic fund protection" guarantee effective until time $\tau$. The guaranteed amount is $L \in(0, S(0)]$. As soon as the investor's account value drops below $L$, the account is immediately credited with just sufficient number of fund units to restore the account value to $L$.

For $t \in[0, \tau]$, let $n(t)$ denote the number of units of the mutual fund in the investor's account at time $t$. As shown in Gerber, Shiu and Yang (2012, Section 7),

$$
n(t)=\max \left\{1, \frac{L}{S(0)} e^{-m(t)}\right\}
$$


Thus, the account value at time $t$ is

$$
\begin{aligned}
n(t) S(t) & =S(t)+[n(t)-1] S(t) \\
& =S(t)+e^{X(t)-m(t)}\left[L-S(0) e^{m(t)}\right]_{+} .
\end{aligned}
$$

To value the "dynamic fund protection" guarantee, we find the expectation

$$
\mathrm{E}\left[e^{X(\tau)-m(\tau)}\left[L-S(0) e^{m(\tau)}\right]_{+}\right]
$$

which, by the independence property discussed in Section 3, is

$$
\begin{aligned}
\mathrm{E}\left[e^{X(\tau)-m(\tau)}\right] \times \mathrm{E}\left[\left[L-S(0) e^{m(\tau)}\right]_{+}\right] & =\mathrm{E}\left[e^{M(\tau)}\right] \times \mathrm{E}\left[\left[L-S(0) e^{m(\tau)}\right]_{+}\right] \\
& =M_{M(\tau)}(1) \sum_{j=1}^{m+1} a_{j}^{*} \eta\left(\alpha_{j} ; L\right)
\end{aligned}
$$

which is similar to $(7.5)$.

\section{Dynamic withdrawal benefit with guarantee}

In this section, we consider a model "dual" to the one in the last section. Here, $L \geq S(0)$. An investor has one unit of a mutual fund at time 0 . If his account value ever goes above $L$, the excess will be immediately paid back to him as "dividends". Ko, Shiu and Wei (2010) use the term dynamic withdrawal benefit to describe such a payoff feature. Again, let $n(t)$ denote the number of units of the mutual fund in the investor's account at time $t$. Then,

$$
n(t)=\min \left\{1, \frac{L}{S(0)} e^{-M(t)}\right\}
$$

which is a formula "dual" to (9.1). In addition to the dividends up to time $\tau$, the investor wants a guarantee that is at least $K, K \leq L$, is to be paid at time $\tau$. That is, the payoff at time $\tau$ is guaranteed to be

$$
\max \{K, n(\tau) S(\tau)\}=n(\tau) S(\tau)+[K-n(\tau) S(\tau)]_{+} .
$$

Our problem is to find the expectation of the last term in (10.2).

Now,

$$
\begin{aligned}
{[K-n(\tau) S(\tau)]_{+} } & =\left[K-\min \left\{S(\tau), L e^{X(\tau)-M(\tau)}\right\}\right]_{+} \\
& =I_{(M(\tau)<\ell)}[K-S(\tau)]_{+}+I_{(M(\tau) \geq \ell)}\left[K-L e^{X(\tau)-M(\tau)}\right]_{+},
\end{aligned}
$$

where $\ell$ is defined by (8.2). The first term on the RHS of (10.3) is the payoff of an up-and-out put option, whose expectation can be found by using results in Section 8 . The expectation of the second term, by the independence property, is the product of two expectations,

$$
\mathrm{E}\left[I_{(M(\tau) \geq \ell)}\right] \mathrm{E}\left[\left[K-L e^{X(\tau)-M(\tau)}\right]_{+}\right]=\operatorname{Pr}[M(\tau) \geq \ell] \mathrm{E}\left[\left[K-L e^{m(\tau)}\right]_{+}\right] .
$$




\section{Concluding remarks}

A main goal of this paper is to calculate or estimate expected discounted values of the form (1.5), where the stock price process is the exponential of a Brownian motion plus an independent compound Poisson process whose upward and downward jumps are modeled by combinations (or mixtures) of exponential distributions. Although $T_{x}$ is the symbol for the time until death random variable, nothing in the paper need to be changed if it is a time until catastrophe random variable or just some positive random variable that is independent of the stock price process. The formulas that we have derived do not depend on whether $\delta$ is risk-free or risk-adjusted or whether the probability measure is risk-neutral or actual.

For finding a combination of exponential distributions to approximate the distribution of $T_{x}$,

$$
f_{T_{x}}(t) \approx \sum_{j} c_{j} f_{\tau_{j}}(t)
$$

two papers by Dufresne (2007a, 2007b) are helpful. From (11.1) and (1.8), we have

$$
\mathrm{E}\left[e^{-\delta T_{x}} g_{T_{x}}(S)\right] \approx \sum_{j} c_{j} \frac{\lambda_{j}}{\lambda_{j}+\delta} \mathrm{E}^{*}\left[g_{\tau_{j}}(S)\right],
$$

where the asterisk signifies that the parameter of the exponential random variable $\tau_{j}$ is changed from $\lambda_{j}$ to $\lambda_{j}+\delta$. For actuarial valuation, Kou's model $(m=n=1)$ seems sufficient. For each $\lambda_{j}$, we solve the Cramér-Lundberg equation

$$
D z^{2}+\mu z-\nu \frac{z}{v+z}+\omega \frac{z}{w-z}=\lambda_{j}+\delta
$$

which is equivalent to a quartic equation. As shown in (2.15), there are two positive roots and two negative roots. With a computer, the roots can be readily found. For a check, calculate the product of the four roots and verify that it equals $\left(\lambda_{j}+\delta\right) v w / D$.

Our suggestion of using knock-out options to model lapses and surrenders is perhaps new. We hope to obtain empirical data to refine the model.

Acknowledgements. We would like to thank Hansjoerg Albrecher, Ning Cai, Eric C. K. Cheung, François Dufresne, Denglin Zhou, and the participants of the Nankai Conference on Actuarial Risk and Related Topics and the anonymous referees for their valuable comments. We acknowledge with thanks the support from the Principal Financial Group Foundation, Research Grants Council of the Hong Kong Special Administrative Region (project No. HKU 706611P), and Society of Actuaries' Center of Actuarial Excellence Research Grant.

\section{References}

Albrecher, H., Gerber, H.U., Yang, H., 2010. A direct approach to the discounted penalty function. North American Actuarial Journal 14 (4), 420 - 434, Discussions, 434 - 
447.

Asmussen, S., Avram, F., Pistorius, M.R., 2004. Russian and American put options under exponential phase-type Lévy models. Stochastic Processes and Their Applications 109, 79 - 111.

Avanzi, B., Gerber H.U., 2008. Optimal dividends in the dual model with diffusion. ASTIN Bulletin 38(2), 653 - 667.

Bertoin, J., 1996. Lévy Processes, Cambridge University Press.

Bowers, N., Gerber, H.U., Hickman, J., Jones, D., Nesbitt, C., 1997. Actuarial Mathematics, 2nd edition, Schaumburg, Ill.: Society of Actuaries.

Cai, N., Kou, S.G., 2011. Option pricing under a mixed-exponential jump diffusion model. Management Science, 57 (11), 2067 - 2081.

Chan, B., 1990. Ruin probability for translated combination of exponential claims. ASTIN Bulletin 20(1), 113 - 114.

Chan, B., Gerber, H.U., Shiu, E.S.W., 2006. Discussion of "On a classical risk model with a constant dividend barrier," North American Actuarial Journal 10(2), 133 139 .

Doney, R.A., 2007. Fluctuation Theory of Lévy Processes, Lecture Notes in Mathematics, Vol. 1897. Springer.

Dufresne, D., 2007a. Fitting combinations of exponentials to probability distributions. Applied Stochastic Models in Business and Industry 23, 23 - 48.

Dufresne, D., 2007b. Stochastic life annuities. North American Actuarial Journal 11 (1), $136-157$.

Dufresne, F., Gerber, H. U., 1988. The probability and severity of ruin for combinations of exponential claim amount distributions and their translations. Insurance: Mathematics and Economics 7(2), 75 - 80.

Dufresne, F., Gerber, H. U., 1989. Three methods to calculate the probability of ruin. ASTIN Bulletin 19(1), 71 - 90.

Dufresne, F., Gerber, H. U., 1991a. Rational ruin problems - a note for the teacher. Insurance: Mathematics and Economics 10(1), 21 - 29.

Dufresne, F., Gerber, H. U., 1991b. Risk theory for the compound Poisson process that is perturbed by diffusion. Insurance: Mathematics and Economics 10(1), 51 - 59.

Gerber, H. U., 1970. An extension of the renewal equation and its application in the collective theory of risk. Scandinavian Actuarial Journal 205 - 210.

Gerber, H. U., 1972. Games of economic survival with discrete- and continuous-income processes. Operations Research 20(1), 37 - 45. 
Gerber, H. U., Shiu, E. S., 1998. On the time value of ruin. North American Actuarial Journal 2 (1), 48 - 71.

Gerber H. U., Shiu E. S. W., 2005. The time value of ruin in a Sparre Andersen model. North American Actuarial Journal 9(2), 49 - 84.

Gerber, H. U., Shiu, E. S. W., Smith, N., 2006. Maximizing dividends without bankruptcy. ASTIN Bulletin 36(1), 5 - 23.

Gerber, H.U., Shiu, E.S.W., Yang, H., 2012. Valuing equity-linked death benefits and other contingent options: a discounted density approach. Insurance: Mathematics and Economics 51 (1), 73 - 92.

Ko, B., Ng, A.C.Y., 2007. Discussion on "Stochastic life annuities" by D. Dufresne. North American Actuarial Journal 11 (3), 170-171.

Ko, B., Shiu, E.S.W., Wei, L., 2010. Pricing maturity guarantee with dynamic withdrawal benefit. Insurance: Mathematics and Economics 47 (2), 216-223.

Kou, S.G., 2008. Jump-diffusion models for asset pricing in financial engineering. In Birge, J. R., Linetsky, V. (Eds.). Handbooks in Operations Research and Management Science, Vol. 15. Elsevier, 73 - 115.

Kyprianou, A.E., 2006. Introductory Lectures on Fluctuations of Lévy Processes with Applications. Springer.

Merton, R. C., 1976. Option pricing when underlying stock returns are discontinuous. Journal of Financial Economics 3(1), 125 - 144.

Mordecki, E., 2002. The distribution of the maximum of a Lévy process with positive jumps of phase-type. Theory of Stochastic Processes, 8(24), 309-316.

Samuelson, P. A., 1965. Rational theory of warrant pricing. Industrial Management Review 6, 13 - 31 .

Täcklind, S., 1942. Sur le risque de ruine dans des jeux inéquitables. Skandinavisk Aktuariedtiskrift 25, 1 - 42. 